\title{
Desenvolvimento de alpínia sob diferentes telas de sombreamento e espaçamentos de cultivo ${ }^{(1)}$
}

\author{
ROSEANE RODRIGUES DE SOUZA(2), ÂNGELA MARIA PEREIRA NASCIMENTO(2), \\ PATRÍCIA DUARTE DE OLIVEIRA PAIVA(2), ELKA FABIANA APARECIDA ALMEIDA ${ }^{(3)}$, \\ PAULO ROBERTO CORREA LANDGRAF(4)
}

\begin{abstract}
RESUMO
A intensidade e a qualidade espectral da radiação podem ser manipuladas com o uso de malhas de sombreamento neutras ou fotoconversoras no cultivo de plantas, podendo promover mudanças fisiológicas e morfológicas. Desse modo, objetivou-se avaliar os efeitos de diferentes níveis de sombreamento, qualidade espectral de malhas e espaçamentos no desenvolvimento de alpínia cv. Jungle King e cv. Jungle Queen. Rizomas foram plantados em 5 ambientes de cultivo (pleno sol, malha preta 30\% de sombreamento, malha preta $50 \%$ de sombreamento, malha azul $50 \%$ de sombreamento e malha vermelha $50 \%$ de sombreamento) e 2 espaçamentos $(0,8 \times 1,0 \mathrm{~m}$ e $0,8 \times 1,5 \mathrm{~m})$. O delineamento experimental foi inteiramente casualizado em esquema fatorial $5 \times 2 \times 2 \times 7$, com os 5 ambientes de cultivo, 2 cultivares, 2 espaçamentos e 7 tempos de avaliação $(55,120,200,255,285$, 335 e 370 dias após o plantio (DAP)). As plantas foram avaliadas quanto ao índice de brotação, número de brotos por planta, altura dos brotos e número de folhas por broto. A brotação da alpínia cv. Jungle Queen ocorreu mais precocemente comparando-se à cv. Jungle King. Não houve influência do espaçamento no desenvolvimento inicial das plantas, mas o efeito das telas de sombreamento foi evidenciado pelo melhor desenvolvimento em altura das plantas quando cultivadas sob sombreamento de $50 \%$ azul ou vermelha.
\end{abstract}

Palavras-chave: Alpinia purpurata, radiação solar, plantas tropicais.

\begin{abstract}
Spectral quality of the shading and spacing on alpinia cultivation

Intensity and spectral quality of the radiation can be manipulated with the use of neutral or color shading screens in growing plants, and it can promote physiological and morphological changes. Therefore, the purpose was evaluating the effects of different shading levels, screens spectral quality, and spacing in the development of cv. Jungle King and cv. Jungle Queen gingers. Rhizomes were planted in 5 cultivation environments (full sun, black screen $50 \%$, black screen $30 \%$, blue screen $50 \%$ and red screen $50 \%$ shading) and 2 spacing $(0.8 \times 1.0 \mathrm{~m}$ and $0.8 \times 1.5 \mathrm{~m})$. The experimental design was completely randomized in a factorial $5 \times 2 \times 2 \times 7$, with 5 environments of cultivation, 2 cultivars, 2 spacing between plants and 7 times of evaluation (55, 120, 200, 255, 285, 335 e 370 days after planting (DAP)). The evaluations were shooting index, number of shoots per plant, shoot height and number of leaves per shoot. Shooting of cv. Jungle Queen was earlier compared to cv. Jungle King. Spacing did not influence in the initial development of plants. Screens with $50 \%$ shading, in blue or red colors, enhanced alpinia development.
\end{abstract}

Keywords: Alpinia purpurata, solar radiation, tropical plants.

\section{INTRODUÇÃO}

Dentre as espécies de flores tropicais, a alpínia (Alpinia purpurata) se destaca devido ao grande potencial produtivo, no entanto não há informações precisas sobre condições de cultivo em algumas regiões, sobretudo aquelas mais promissoras (LANDGRAF e PAIVA, 2009a, LANDGRAD e PAIVA, 2009b).

A intensidade e qualidade da radiação no ambiente de cultivo, como das malhas de sombreamento, podem modificar o microclima e influenciar mudanças fisiológicas e morfológicas vegetais (LIMA et al., 2010), além de processos normalmente considerados como os aspectos nutricionais (HERNANDEZ et al., 2014). A manipulação das propriedades da radiação pode ser realizada com uso de malhas neutras ou fotoconversoras, em diferentes níveis de sombreamento (KITTAS et al., 1999).
Plantas ornamentais sensíveis à incidência direta da radiação solar são cultivadas comercialmente sob sombreamento parcial proporcionado por malhas, sendo as pretas as mais usadas. Essas malhas transmitem a faixa do espectro visível da radiação uniformemente, atuando como filtros de densidade neutra. Ao contrário, as malhas fotoconversoras combinam proteção física e filtragem diferencial da radiação, em que a intensidade e a qualidade espectral transmitida são alteradas, com mudanças ópticas da dispersão e reflectância (OREN-SHAMIR et al., 2001; SHAHAK et al., 2004).

Crescimento mais lento foi observado por OrenShamir et al. (2001) em plantas de pitósporo (Pittosporum variegatum) cultivadas sob malha azul, com inibição da taxa de alongamento, redução da ramificação lateral, encurtamento do internódio e diminuição da produtividade de ramos comerciais, no entanto, esses autores observaram

\footnotetext{
(1) Recebido em 21/11/2015 e aceito em 08/09/2016

(2) Universidade Federal de Lavras (UFLA), Departamento de Agricultura, Lavras-MG, Brasil. *Autor correspondente: angela mpn2@yahoo.com.br

(3) Universidade Federal de Minas Gerais (UFMG), Montes Claros-MG, Brasil

(4) Universidade de Alfenas (UNIFENAS), Alfenas-MG, Brasil
} 
que plantas cultivadas sob malha vermelha apresentaram maior alongamento, ramificação e comprimento de internódio. Cultivares e híbridos de orquídea Phalaenopsis tiveram sua área e biomassa foliar incrementadas em cultivo sob malha azul enquanto que plantas cultivadas sob malha vermelha floresceram precocemente (LEITE et al., 2008).

O espaçamento de plantio também interfere na produtividade e na qualidade das flores de corte. O plantio mais adensado tende a aumentar a produção de hastes, no entanto, o tamanho dessas pode não atingir o padrão comercial. Por outro lado, o plantio mais espaçado promove a produção de hastes de melhor qualidade, pois as plantas possuem maior disponibilidade de espaço, água e nutrientes para o seu desenvolvimento. O espaçamento recomendado pode variar de 1,0 a 2,0 m entre plantas e 1,5 a 3,0 m entre linhas (TEIXEIRA e LOGES, 2012). Em observações de Bezerra et al. (2008), alpínias com dois anos de cultivo em espaçamento mais adensado $(0,65 \times 2,00 \mathrm{~m})$ aumentaram a emissão de hastes, contudo, espaçamentos maiores $(0,90 \mathrm{x}$ $2,00 \mathrm{~m}$ e 1,25 x 2,00 m) promoveram a produção de hastes mais longas.

Desse modo, objetivou-se avaliar os efeitos de diferentes níveis de sombreamento, qualidade espectral de malhas e espaçamentos no desenvolvimento de alpínias cv. Jungle King e cv. Jungle Queen.

\section{MATERIAL E MÉTODOS}

O experimento foi instalado no município de Lavras, localizado na região Sul de Minas Gerais, a $918 \mathrm{~m}$ de altitude, $21^{\circ} 14^{\prime}$ de latitude sul e $45^{\circ} 00^{\prime}$ de longitude oeste. Na classificação climática de Köppen, o clima enquadrase no tipo Cwa, apresentando duas estações definidas: seca com temperaturas mais baixas, de abril a setembro, e chuvosa, com temperaturas mais elevadas, de outubro a março (DANTAS et al., 2007). As temperaturas médias mensais em Lavras (MG), referentes ao período de avaliação do experimento, de abril de 2011 a abril de 2012, foram fornecidas pela Estação Climatológica pertencente ao $5^{\circ}$ Distrito de Meteorologia do INMET em convênio com a UFLA.
Rizomas de Alpinia purpurata 'Jungle Queen' e 'Jungle King', obtidos de uma produção comercial, foram desinfestados em solução de hipoclorito de sódio $0,2 \mathrm{~g} \mathrm{~L}^{-1}$ por $20 \mathrm{~min}$. e, após a secagem desses, mensurou-se o diâmetro, cujas médias foram de $1,66 \mathrm{~cm}$ para 'Jungle Queen' e 5,38 cm para 'Jungle King'.

O plantio dos rizomas foi realizado em abril de 2011, em covas de $30 \times 30 \mathrm{~cm}$ e adubação inicial com $2,5 \mathrm{~kg}$ $\mathrm{m}^{-2}$ de esterco de curral curtido e $50 \mathrm{~g}$ do formulado NPK 10:10:10. O solo da área experimental, classificado como Latossolo Vermelho Distrófico, apresentava-se no plantio com fertilidade média na camada de 0 a 20 $\mathrm{cm}$, com pH de 5,6, matéria orgânica de 4,4 dag kg-1 e saturação por bases de 39,2\%. Aos 60 dias após o plantio (DAP), as plantas receberam adubação de cobertura com 10 g planta $^{-1}$ de NPK 10:10:10. Adubações de cobertura com $1 \mathrm{~kg} \mathrm{~m}^{-2}$ de esterco de curral curtido e $30 \mathrm{~g}$ de Yoorin ${ }^{\circledR}$ Master por planta foram feitas aos 250 e 340 DAP. A área plantada foi irrigada por microaspersão e o controle de plantas infestantes foi feito por capinas manuais.

O delineamento experimental utilizado foi inteiramente casualizado, em esquema fatorial $5 \times 2 \times 2$ x 7. Foram testados 5 ambientes de cultivos (pleno sol, malha preta $30 \%$ de sombreamento, malha preta $50 \%$ de sombreamento, malha azul 50\% de sombreamento e malha vermelha $50 \%$ de sombreamento), 2 espaçamentos de cultivo $(0,8 \times 1,0 \mathrm{~m}$ e $0,8 \times 1,5 \mathrm{~m}), 2$ cultivares (Jungle Queen e Jungle King) e 7 tempos de avaliação (55, 120, $200,255,285,335$ e 370 dias após plantio (DAP)), com 4 repetições e 2 plantas por parcela.

As plantas foram observando-se o índice de brotação, número de brotos por planta, altura dos brotos $(\mathrm{cm})$ e número de folhas por broto. Os dados obtidos foram submetidos à análise de variância e os dados ajustados a uma curva de regressão polinomial com o auxílio do programa estatístico SISVAR 4.6 (FERREIRA, 2011).

\section{RESULTADOS E DISCUSSÃO}

As médias de temperatura observadas durante o período experimento são apresentadas na tabela 1 .

Tabela 1. Temperaturas médias mensais observadas no período abril de 2011 a abril de 2012.

Table 1. Monthly average temperatures observed in the period April 2011 to April 2012.

\begin{tabular}{|c|c|c|c|c|c|c|c|c|c|c|c|c|c|}
\hline & \multicolumn{9}{|c|}{2011} & \multicolumn{4}{|c|}{2012} \\
\hline Mês & Abr & Mai & Jun & Jul & Ago & Set & Out & Nov & Dez & Jan & Fev & Mar & Abr \\
\hline $\begin{array}{c}\text { Temperatura } \\
\text { média }\left({ }^{\circ} \text { C) }\right.\end{array}$ & 21,3 & 18,3 & 16,3 & 17,3 & 19,6 & 20,2 & 20,9 & 20,4 & 22,2 & 21,4 & 23,1 & 22,3 & 21,4 \\
\hline
\end{tabular}

Fonte: Estação Climatológica pertencente ao $5^{\circ}$ Distrito de Meteorologia do INMET -UFLA

Analisando-se o índice de brotação dos rizomas, foi observada interação entre as cultivares e tempo de avaliação. O índice de brotação foi de $77,1 \%$ para o cultivar Jungle King e 79,3\% para cultivar Jungle Queen após 370 dias de plantio, sendo influenciado pela elevação das temperaturas médias mensais (Tabela 1), independente da cultivar (Figura 1), uma vez que a produção de alpínias é favorecida por temperaturas mais elevadas (TERAO et al., 2005). 


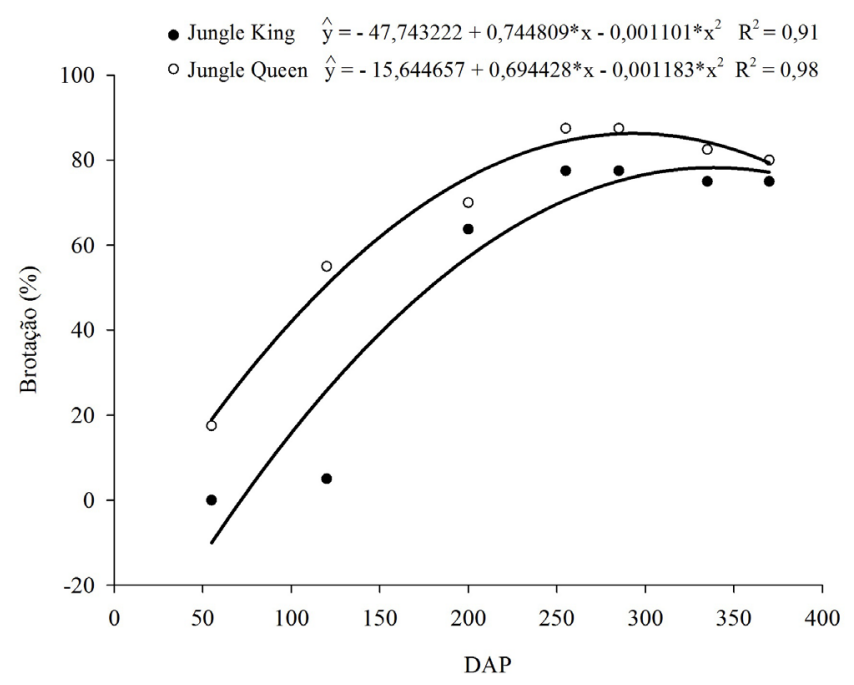

Figura 1. Brotação dos rizomas de alpínia cv. Jungle King e cv. Jungle Queen, em função dos dias após o plantio (DAP). Figure 1. Shoot growth of alpinia rhizomes cv. Jungle King and cv. Jungle Queen, as a function of days after planting.

A emissão de brotos iniciou-se aos 107,8 dias após o plantio para o cultivar Jungle Queen e aos 112,1 dias para cultivar Jungle King. Observa-se ainda que, a partir de 250 dias, há tendência de estabilização na emissão de novas brotações. Para o número de brotos formados por planta também foi observada interação entre cultivares e tempo de avaliação. O cultivar Jungle Queen apresentou 11,4 brotos por planta, aos 370 DAP, comparando-se com a média de 8,9 brotos por planta do cultivar Jungle King, no mesmo período (Figura 2). Diferenças entre cultivares relacionadas à velocidade e número de brotos também foram observadas em bastão-do-imperador (NASCIMENTO et al., 2015).

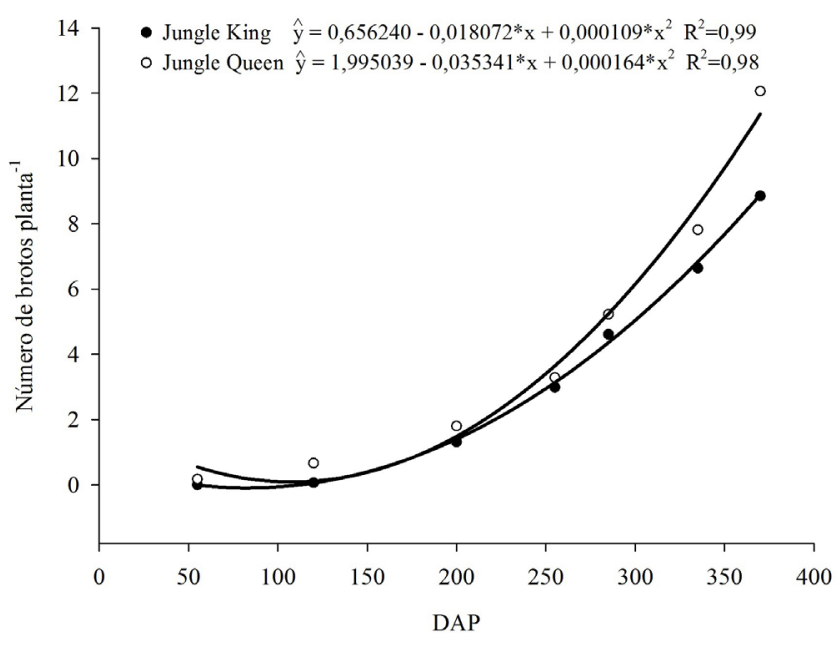

Figura 2. Número de brotos por planta de alpínia cv. Jungle King e cv. Jungle Queen, e $\mathrm{m}$ função dos dias após o plantio (DAP).

Figure 2. Number of shoots per plant of alpinia cv. Jungle King and cv. Jungle Queen, as a function of days after planting. 
A brotação dos rizomas teve início a partir dos 107 DAP, independente do cultivar, podendo-se inferir que houve um período de dormência desses rizomas, visto que o plantio foi realizado no outono, quando a temperatura começou a diminuir. Com o aumento da temperatura, a partir dos meses de agosto e setembro, correspondente ao final do inverno e início da primavera, iniciou-se a brotação (Figura 2). A ocorrência de dormência em rizomas de zingiberáceas pode ocorrer em regiões frias ou com inverno mais rigoroso (DEMMY e BURCH, 1998; NASCIMENTO et al, 2015).

Analisando-se o número de folhas formadas, foi observada interação entre o tempo de avaliação e as cultivares, sendo que os cultivares Jungle Queen e Jungle King apresentaram tendências semelhantes de desenvolvimento em função do tempo (Figura 3).

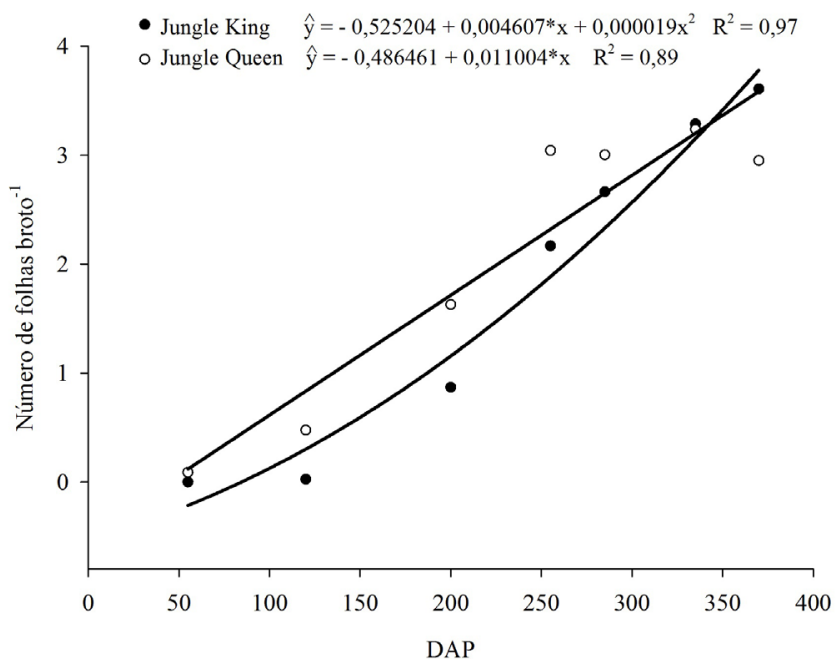

Figura 3. Número de folhas de alpínia cv. Jungle King e cv. Jungle Queen, em função dos dias após o plantio (DAP).

Figure 3. Number of leaves of alpinia cv. Jungle King and cv. Jungle Queen, as a function of days after planting.

Para altura das plantas foi observada interação entre os diferentes ambientes de cultivo e o tempo de avaliação. Em relação à influência das malhas de sombreamento, os brotos desenvolvidos de plantas cultivadas sob malha fotoconversora vermelha, que emitem em maior intensidade comprimentos de onde vermelho, apresentaram maior incremento em altura, seguido dos brotos desenvolvidos de rizomas cultivados sob malha azul (comprimento de onda azul), sendo superiores ao cultivo sob malhas pretas (Figura 4). Foi observado aumento na altura de dracenas sob malha vermelha (KOBAYASHI et al., 2006) e de crisântemos sob malha azul (OYAERT et al., 1999).

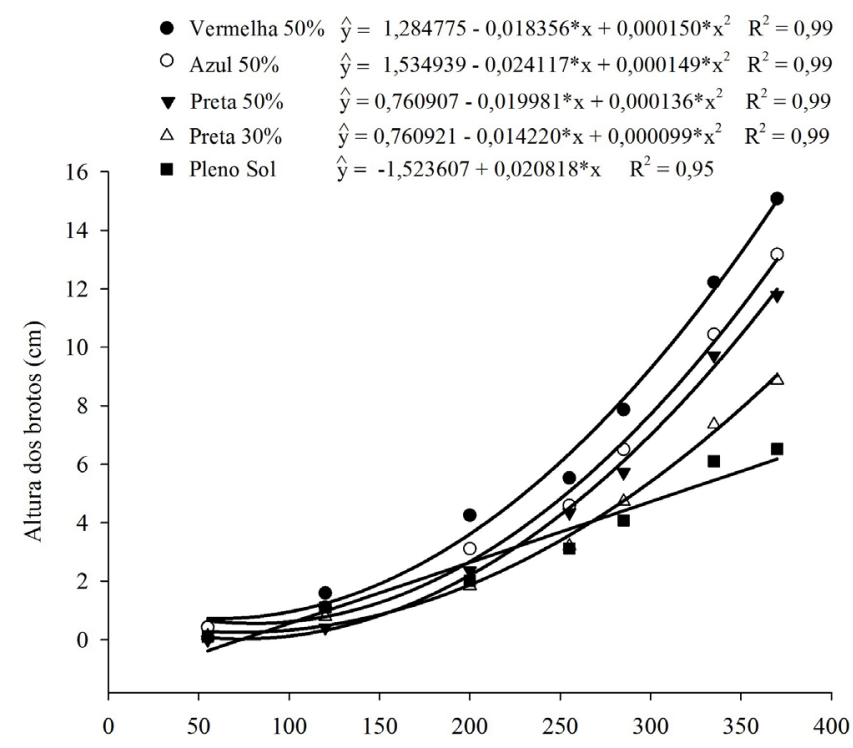

Figura 4. Altura dos brotos de plantas de alpínia cv. Jungle King e cv. Jungle Queen cultivadas sob diferentes telas de sombreamentos, em função dos dias após o plantio (DAP).

Figure 4. Height of alpinia shoots. cv. Jungle King and cv. Jungle Queen grown under different shading screens according to the days after planting (DAP). 
O crescimento em altura dos brotos sob os diferentes ambientes de cultivo foi semelhante até os 200 DAP. A elevação nas temperaturas médias após esse período contribuiu para o aumento na altura das alpínias, principalmente em plantas cultivadas sob malhas com 50\% de sombreamento, sendo que esse nível de sombreamento promoveu maior incremento em altura comparada à malha $30 \%$ e ao cultivo a pleno sol. Aos 370 DAP, a altura média dos brotos cultivados sob malha vermelha foi 2,3 vezes maior em relação à dos brotos cultivados a pleno sol, indicando não ser favorável o cultivo nessa condição.

Também não houve desenvolvimento satisfatório quando cultivados sob $30 \%$ de sombreamento em malha negra, contradizendo Lamas (2004), que indicam a possibilidade de desenvolvimento dessas plantas sob sombreamento de 20 a $45 \%$.

Em relação ao efeito de telas fotoconversoras no desenvolvimento em espécies ornamentais, resultados antagônicos foram observados, pois, ao contrário de Meirelles et al. (2007), que não observaram diferença na altura de mudas de Rhapis excelsa cultivadas sob diferentes malhas de sombreamento e de Oren-Shamir et al. (2001) que observaram redução no desenvolvimento de pitósporo cultivado sob malha azul, em alpínias pode-se constatar maior altura de plantas mantidas sob telas vermelhas e azul. Também em Dracaena marginata 'Colorama', cultivada sob malha vermelha, verificou-se aumento em altura e no número de folhas (KOBAYASHI et al., 2006). Mas, ao contrário, o uso de malha preta proporcionou melhores condições para o crescimento vegetal e a produção de hastes florais de Anthurium andraeanum 'Apalai' (NOMURA et al., 2009; LIMA et al., 2010) e para Bauhinia variegata, as telas não apresentaram efeito (MAZZINI-GUEDES e PIVETTA, 2014). Além do desenvolvimento, o uso de telas de coloridas pode afetar características anatômicas, como a densidade estomática de acordo com observações de Silva et al. (2015).

A brotação tardia das cultivares de alpínia, associada à época da realização do plantio (outono), não estimulou um perfilhamento mais intenso. Desse modo, os espaçamentos de plantio utilizados não influenciaram no desenvolvimento inicial das plantas.

Em relação à tonalidade verde das folhas de alpínia, em ambas os cultivares, observou-se, visualmente, que plantas cultivadas a pleno sol possuíam folhas em um tom de verde mais claro. Ao contrário, as folhas das plantas crescidas sob malhas preta, azul e vermelha apresentaram tons verdeescuros. A incidência direta da radiação solar sob as alpínias cultivadas a pleno sol pode ter provocado algum dano em consequência do excesso de radiação recebido pelas plantas.

\section{CONCLUSÕES}

- Recomenda-se que o cultivo de alpínias seja feito sob malhas fotoconversoras vermelha ou azul, com 50\% de sombreamento.

- Não se observou influência dos espaçamentos de plantio testados no desenvolvimento inicial das plantas.

- Nas condições experimentais avaliadas o cultivar Jungle Queen apresentou brotação precoce em relação ao cultivar Jungle King.

\section{AGRADECIMENTOS}

Os autores agradecem ao CNPq e FAPEMIG pelo financiamento do projeto e concessão de bolsas.

\section{REFERÊNCIAS}

BEZERRA, F.C. GONDIM, R.S.; PEREIRA, N.S. Produção de alpínia em cultivo protegido na região litorânea do estado do Ceará. Fortaleza: Embrapa Agroindústria Tropical, 2008, 4p. (Comunicado técnico, 137).

DANTAS, A.A.A.; CARVALHO, L.G.; FERREIRA, E. Classificação e tendências climáticas em Lavras, MG. Ciência e Agrotecnologia, v.31, n.6, p.1862-1866, 2007.

DEMMY, E.W.; BURCH, D. Gingers: a rising star in Florida gardens. Proceedings of the Florida State Horticultural Society, v.111, p.190-191, 1998.

FERREIRA, D.F. Sisvar: a computer statistical analysis system. Ciência e Agrotecnologia, v.35, n.6, p.1039-1042, 2011.

HERNADEZ, M.S.; ALVAREZ, R.G.; RIVERA-CRUZ, M.C.; ÁLVAREZ-SOLIS, J.D.; PAT-FERNANDES, J.M.; ORTIZ-GARCIA, C.F. The influence of organic fertilizers ont he chemical properties of soil and the production of Alpinia purpurata. Ciencia e Investigacíon Agraria, v.41, n.2, p.215-224, 2014.

KITTAS, C. BAILLE, A.; GIAGLARAS, P. Influence of covering material and shading on the spectral distribution of light in greenhouses. Journal of Agricultural Engineering Research, v.73, n.4, p.341-351, 1999.

KOBAYASHI, K.D.; KAWABATA, A.; LICHTY, J.S. Effects of photoselective shadecloths on potted Dracaena and Anthurium plants. HortScience, v.41, n.4, p.10531054, 2006.

LAMAS, A.M. Floricultura tropical: técnicas de produção. 2004, 65p.

LANDGRAF, P.R.C.; PAIVA, P.D.O. Produção de flores cortadas no estado de Minas Gerais. Ciência e Agrotecnologia, v.33, n.1, p.120-126, 2009.

LANDGRAF, P.R.C.; PAIVA, P.D.O. Produção de mudas para jardim no estado de Minas Gerais. Ciência e Agrotecnologia, v.33, n.1, p.127-131, 2009.

LEITE, C.A. ITO, R.M.; LEE, G.T.S.; GANELEVIN, R.; FAGNANI, M.A. Light spectrum management using colored nets to control the growth and blooming of Phalaenopsis. Acta Horticulturae, n.770, p.177-184, 2008. 
LIMA, J.D.; NOMURA, E.S.; FUZITANI, E.J.; SILVA, S.H.M.G. Variáveis fisiológicas de antúrio cultivado sob diferentes malhas de sombreamento. Scientia Agraria, v.11, n.3, p.193-200, 2010.

MAZZINI-GUEDES, R.B.; PIVETTA, K.F.L. Initial growth of Baunhinia variegata trees under different colored shade nets and light conditions. Revista Árvore, v.38, n.6, p.1133-1145, 2014.

MEIRELLES, A.J.A.; PAIVA, P.D.O.; OLIVEIRA, M.I.; TAVARES, T.S. Influência de diferentes sombreamentos e nutrição foliar no desenvolvimento de mudas de palmeira ráfia Rhapis excelsa (Thunberg) Henry ex. Rehder. Ciência e Agrotecnologia, v.31, n.6, p.1884-1887, 2007.

NASCIMENTO, A.M.P; PAIVA, P.D.O.; NERY, F.C.; SOUZA, R.R.; MANFREDINI, G.M.; ALMEIDA, E.F.A. Revista Brasileira de Ciências Agrárias, v.10, n.2, p.230236, 2015.

NOMURA, E.S. LIMA, J.D.; RODRIGUES, D.S.; GARCIA, V.A.; FUZITANI, E.J.; SILVA, S.H.M. Crescimento e produção de antúrio cultivado sob diferentes malhas de sombreamento. Ciência Rural, v.39, n.5, p.1394-1400, 2009.

OREN-SHAMIR, M. GUSSAKOVSKY, E.E.; SHPIEGEL, E.; NISSIM-LEVI, A.; RATNER, K.; OVADIA, R.; GILLER, YU.E.; SHAHAK, Y. Coloured shade nets can improve the yield and quality of green decorative branches of Pittosphorum variegatum. The Journal of Horticultural Science and Biotechnology, v.76, n.3, p.353-361, 2001.
OYAERT, E.; VOLKRAERT, E.; DEBERGH, P. Growth of chrysanthemum under coloured plastic films with different light qualities and quantities. Scientia Horticulture, v.79, p.195-205, 1999.

SHAHAK, Y.; GUSSAKOVSKY, E.E.; GAL, E.; GANELEVIN, R. Colornets: crop protection and lightquality manipulation in one technology. Acta Horticulture, n.659, p.143-151, 2004.

SILVA, R.A.L.; SOARES, J.D.R.; DIAS, G.M.G.; PASQUAL, M.; CHAGAS, E.A.; GAVILANES, M.L. Cultivo de tamarindo sob malhas coloridas: plasticidade anatômica foliar. Ciência Rural, v.45, n.2, p.238-244, 2015.

TEIXEIRA, M.C.F.; LOGES, V. Alpinia. In: PAIVA, P.D.O; ALMEIDA, E.F.A. Produção de flores de corte. Lavras: Editora UFLA, 2012, p.40-57.

TERAO, D.; CARVALHO, A.C.C.P.; BARROSO, T.C.F.S. Flores tropicais. Brasília: Embrapa Informação Tecnológica, 2005. 225p. 